\title{
Oxidation of Thiol Using Ionic Liquid-Supported Organotelluride as a Recyclable Catalyst
}

\author{
Aya Mihoya ${ }^{1}$, Shinichi Koguchi ${ }^{1, *}$, Yuga Shibuya ${ }^{1}$, Minato Mimura ${ }^{1}$ and Makoto Oba ${ }^{2, *}$ \\ 1 Department of Chemistry, Tokai University, 4-1-1 Kitakaname, Hiratsuka, Kanagawa 259-1292, Japan; \\ 7btad011@mail.u-tokai.ac.jp (A.M.); 8bskm003@mail.u-tokai.ac.jp (Y.S.); 6bsc3214@mail.u-tokai.ac.jp (M.M.) \\ 2 Graduate School of Science and Technology, Tokai University, 3-20-1 Orido, Shimizu-ku, \\ Shizuoka 424-8610, Japan \\ * Correspondence: koguchi@tokai-u.jp (S.K.); moba@tokai-u.jp (M.O.); \\ Tel.: +81-46-358-1211 (ext. 3752) (S.K.); +81-54-334-0411 (ext. 3210) (M.O.)
}

Received: 16 March 2020; Accepted: 3 April 2020; Published: 4 April 2020

\begin{abstract}
Organotellurium compounds are known to be useful oxidation reagents. For developing a recoverable and reusable reagent, this paper describes the use of an ionic liquid (IL) support for the organotellurium reagent and its application as a recyclable catalyst for thiol oxidation. We have successfully prepared a novel diphenyl telluride derivative 5 bearing an imidazolium hexafluorophosphate group in its structure. It is found that the IL-supported diphenyl telluride 5 efficiently catalyzed the aerobic oxidation of various thiols in $[\mathrm{bmim}] \mathrm{PF}_{6}$ solution under photosensitized conditions to provide the corresponding disulfides in excellent yields. The product can be isolated by simple ether extraction. The IL-supported catalyst 5 remaining in the ionic liquid phase can be reused for five successive runs while retaining high catalytic activity ( $97 \%$ yield even in the fifth run).
\end{abstract}

Keywords: ionic liquid; recyclable catalyst; organotellurium compound; aerobic oxidation; thiol; photosensitized oxygenation

\section{Introduction}

Recently, organotellurium oxides have been recognized as useful oxidation reagents [1]. In particular, diaryl telluroxides, tellurones, and aryltellurinic acid derivatives have been identified as versatile and effective oxidants for alcohols, phosphines, thiols, thiocarbonyl compounds, and so on [2-11]. Generally, these oxidation reactions require stoichiometric amounts of organotellurium reagents. From synthetic, economic, and environmental perspectives, the development of a catalytic process is desirable. Herein, we have developed organotelluride-catalyzed oxidation of phosphites to phosphates [12], silanes to silanols [13], and thiols to disulfides [14] while employing aerobic oxygen as a terminal oxidant under photosensitized conditions, where the in situ generation of tellurium oxide species by singlet oxygen oxidation is expected. However, the protocol is not without disadvantages, for instance, the product requires isolation by chromatographic purification, and the organotelluride catalyst is rarely reusable. To overcome these issues, we envisioned to immobilize the organotelluride catalyst on an ionic liquid (IL) support.

IL-supported organic synthesis and catalysis have been extensively studied in recent years [15]. Due to its high polarity, the IL support offers the advantages of easy product isolation and catalyst recycling via simple phase separation. We have previously reported the synthesis of IL-supported 18-crown-6 ether [16], ascorbate-based IL [17], and IL-supported benzyl chloride [18] for Huisgen click chemistry, IL-supported hypervalent iodine reagent [19] for alcohol oxidation, and IL-supported 1,3-dimethylimidazolidin-2-one for halogenation [20]. Herein, we describe the synthesis of IL-supported 
diphenyl telluride as a recoverable and reusable oxidation catalyst. The catalytic activity and recyclability of the reagent are evaluated via aerobic oxidation of thiols under photosensitized conditions. The transformation of thiols to disulfides is of interest from the viewpoint of organic and biological processes.

\section{Results and Discussion}

The IL-supported diphenyl telluride was prepared in the following manner (Scheme 1). Using (4-(hydroxymethyl)phenyl)boronic acid (1) as the starting material, (4(phenyltellanyl)phenyl)methanol (2) was produced in $84 \%$ yield from the coupling reaction with diphenyl ditelluride. Next, (4-(chloromethyl)phenyl)(phenyl)tellane (3) was prepared in $94 \%$ yield via halogenation with thionyl chloride followed by basic hydrolysis. Then, 1-methyl-3-(4-(phenyltellanyl)benzyl)-1H-imidazol-3-ium chloride (4) was obtained in 75\% yield by reacting the compound 3 with methyl imidazole. Since this IL-supported telluride 4 was extremely hygroscopic, the anion was subsequently converted to $\mathrm{PF}_{6}{ }^{-}$to produce hydrophobic IL-supported diphenyl telluride (5) in 96\% yield, which was insoluble in low polarity organic solvents and water.

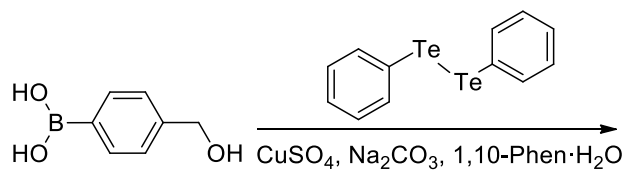

$5 \mathrm{~h}$

(1)

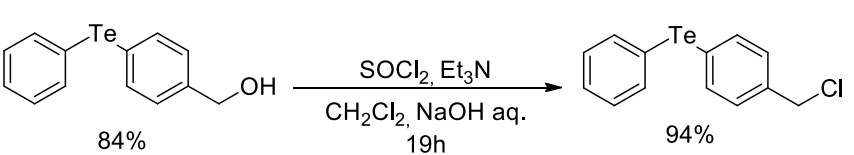

(2)

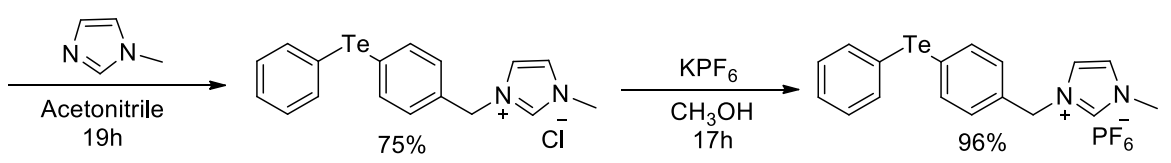

(4)

(5)

Scheme 1. Synthesis of ionic liquid-supported diphenyl telluride.

Initially, we investigated the catalytic oxidation of thiol using IL-supported diphenyl telluride in various ILs employing thiophenol as the model substrate. An IL solution of the thiol, IL-supported catalyst, and rose bengal as a photosensitizer were stirred in an open flask and irradiated with a 500-W halogen lamp. After $3 \mathrm{~h}$, the produced diphenyl disulfide was isolated by extracting with diethyl ether. The yields are compiled in Table 1. The catalytic activities of the IL-supported diphenyl tellurides 4 and 5 were similar to or higher than that of free diphenyl telluride (Entry 7), whereas the reaction was significantly retarded in the absence of the catalyst (Entry 8). The presence of oxygen is also essential for this transformation. In fact, the reaction under nitrogen atmosphere resulted in significant yield reduction (Entry 9). Although the reaction in $[\mathrm{bmim}]\left(\mathrm{CF}_{3} \mathrm{SO}_{2}\right)_{2} \mathrm{~N}$ and $[\mathrm{bmim}] \mathrm{MeSO}_{4}$ reached completion, the isolated yields were slightly lowered owing to the phase separation problems (Entries 1 and 2). The best result was obtained using a hydrophobic $\mathrm{IL}$, [bmim] $\mathrm{PF}_{6}$, as a solvent (Entry 3). Diphenyl disulfide was also isolated in quantitative yields in Entries 4 and 6, however, the IL [bmim] $\mathrm{BF}_{4}$ and the IL-supported catalyst 4 were unsuitable for reuse because of their hygroscopic nature.

Although the active species for this oxidation reaction could not be identified at the present stage, a possible catalytic cycle was proposed according to our previous paper (Figure 1) [14]. Namely, singlet oxygen oxidation of the telluride catalyst 5 gave the corresponding telluroxide (and/or tellurone), which underwent a nucleophilic attack by thiol to afford an adduct $\mathbf{A}$. Then, the adduct $\mathbf{A}$ reacted with another thiol to give the disulfide along with regeneration of the catalyst 5. 
Table 1. Oxidation of thiophenol in the presence of organotellurium catalysts ${ }^{\mathrm{a}}$.
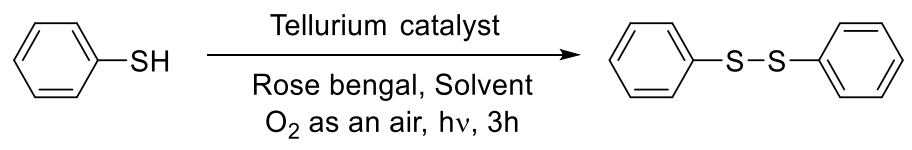

$\mathrm{O}_{2}$ as an air, hv, $3 \mathrm{~h}$

\begin{tabular}{cccc}
\hline Entry & Tellurium Catalyst & Solvent & Yield (\%) \\
\hline 1 & 5 & {$\left[\mathrm{bmim}^{\mathbf{b}}\left(\mathrm{CF}_{3} \mathrm{SO}_{2}\right)_{2} \mathrm{~N}\right.$} & 78 \\
2 & 5 & {$[\mathrm{bmim}] \mathrm{MeSO}_{4}$} & 90 \\
3 & 5 & {$[\mathrm{bmim}] \mathrm{PF}_{6}$} & quant. \\
4 & 5 & {$[\mathrm{bmim}] \mathrm{BF}_{4}$} & quant. \\
5 & 5 & {$[\mathrm{bmim}] \mathrm{Br}$} & 79 \\
6 & 4 & {$[\mathrm{bmim}] \mathrm{PF}_{6}$} & quant. \\
7 & PhTePh & {$\left[\mathrm{bmim}_{6} \mathrm{PF}_{6}\right.$} & 84 \\
8 & none & {$\left[\mathrm{bmim}_{\mathrm{Pm}}\right.$} & 47 \\
$9^{\mathrm{c}}$ & $\mathbf{5}$ & {$[\mathrm{bmim}] \mathrm{PF}_{6}$} & 23 \\
\hline
\end{tabular}

a Condition: thiol $(1 \mathrm{mmol})$, tellurium catalyst $(0.2 \mathrm{mmol})$, rose bengal $(0.05 \mathrm{mmol})$, solvent $(10 \mathrm{~mL})$, irradiated with a 500-W halogen lamp under aerobic conditions, $3 \mathrm{~h}$; ${ }^{\mathrm{b}}$ isolated yield, ${ }^{\mathrm{c}}$ under a nitrogen atmosphere.

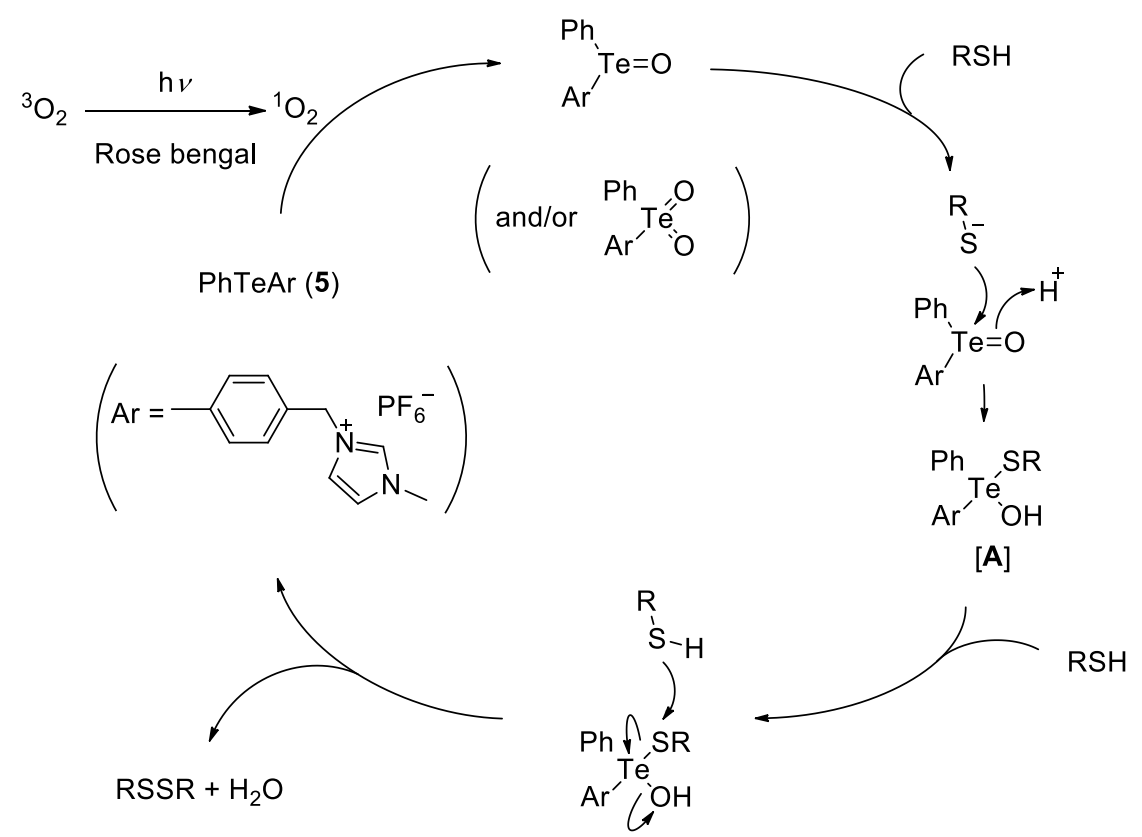

Figure 1. Plausible reaction mechanism for the catalytic oxidation of thiols.

With the optimized conditions in hand, the substrate scope of the oxidation reaction using IL-supported catalyst 5 was evaluated with various thiols. The results are summarized in Table 2. Dodecanethiol (Entry 1), cyclohexanethiol (Entry 2), and benzyl mercaptane (Entry 6) were converted to their corresponding disulfides in high yields. However, the yield of the oxidation reaction of tert-butyl mercaptane decreased to $73 \%$ owing to the steric hindrance (Entry 3). Under the employed oxidation conditions, hydroxyl and ester groups remained untouched to produce their corresponding disulfides in excellent yields (Entries 4 and 5). The reaction also proceeded with the aromatic nitro and chloro functional groups intact (Entries 8 and 9). Furthermore, oxidation of heterocyclic 4-mercaptopyridine produced 4, $4^{\prime}$-dipyridyl disulfide in quantitative yield without affecting the pyridine ring (Entry 10).

Finally, we investigated the reusability of the IL-supported diphenyl telluride 5 employing the conditions shown in Entry 7 of Table 2. After completing the thiophenol oxidation, the resulting diphenyl disulfide was isolated via extraction with diethyl ether, and the remaining $[\mathrm{bmim}] \mathrm{PF}_{6}$ solution containing IL-supported catalyst 5 and rose bengal could be reused at least four times in the subsequent reactions with only a slight decrease in the product yield ( $97 \%$ after four times recycling, Figure 2). 
Table 2. Catalytic oxidation of various thiols using IL-supported diphenyl telluride $5{ }^{\text {a }}$.

$$
\begin{array}{cc}
\mathrm{R}-\mathrm{SH} & \text { IL-supported catalyst } 5 \\
{\mathrm{PF}_{6}} } \\
\mathrm{O}_{2} \text { as an air, hv, } 3 \mathrm{~h}
\end{array} \mathrm{R}-\mathrm{S}-\mathrm{S}-\mathrm{R}
$$

Entry

a Condition: thiol $(0.5 \mathrm{mmol}), \mathbf{5}(0.1 \mathrm{mmol})$, rose bengal $(0.025 \mathrm{mmol}),\left[\mathrm{bmim}_{\mathrm{PF}}(5 \mathrm{~mL})\right.$, irradiated with a $500-\mathrm{W}$ halogen lamp under aerobic conditions, $3 \mathrm{~h} ;{ }^{\mathrm{b}}$ isolated yield.

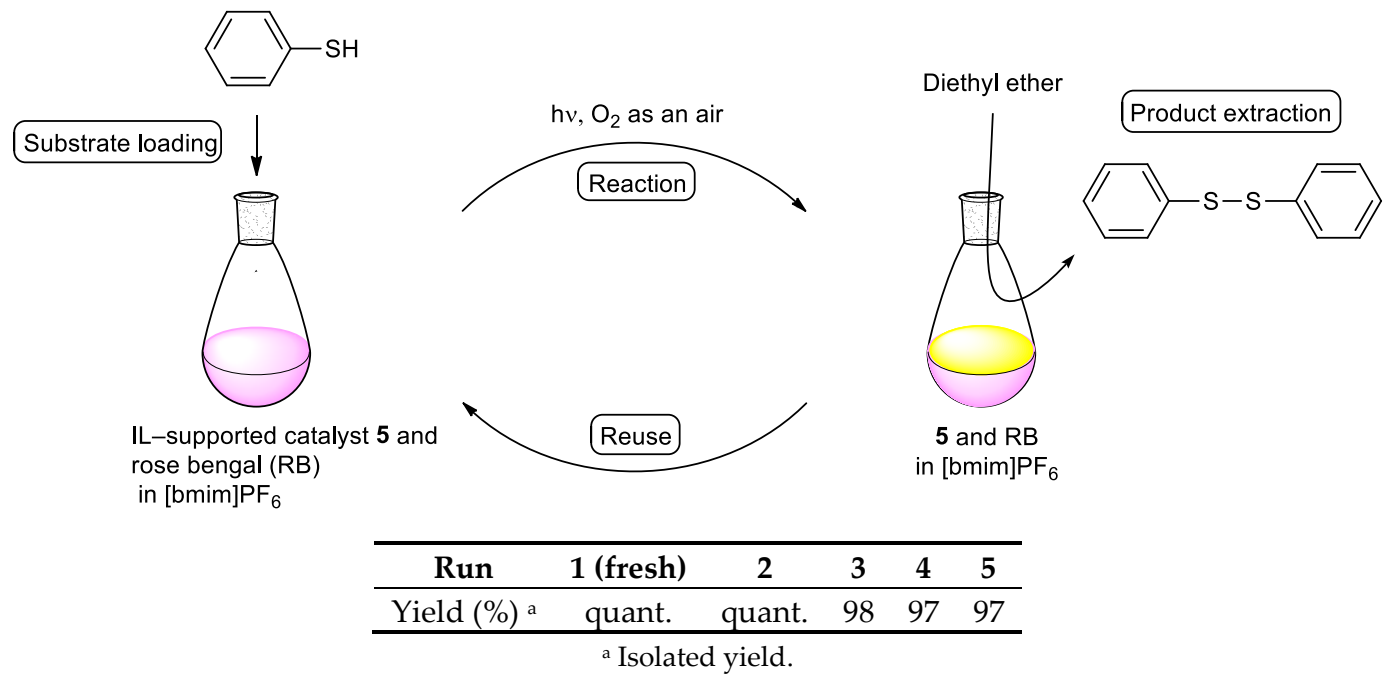

Figure 2. Recycling experiment of IL-supported diphenyl telluride catalyst 5. Condition: thiol (1 mmol), tellurium catalyst $(0.2 \mathrm{mmol})$, rose bengal $(0.05 \mathrm{mmol})$, solvent $(10 \mathrm{~mL})$, irradiated with a $500-\mathrm{W}$ halogen lamp under aerobic conditions, $3 \mathrm{~h}$. 


\section{Materials and Methods}

\subsection{General}

All reagents and solvents were commercially sourced and of reagent grade and were used without purification. The reactions were monitored using aluminum thin layer chromatography plates with silica gel 60 F254 (Merck, (Darmstadt, Germany)). Column chromatography was performed using silica gel 60 (Kanto Chemical, Japan, Tokyo). ${ }^{1} \mathrm{H},{ }^{13} \mathrm{C}$, and ${ }^{125} \mathrm{Te}$ NMR spectra were measured on a Bruker Advance DRX 500 ( ${ }^{1} \mathrm{H}: 500 \mathrm{MHz},{ }^{13} \mathrm{C}: 125 \mathrm{MHz},{ }^{125} \mathrm{Te}: 159 \mathrm{MHz}$ spectrometer. All chemical shifts are reported in parts per million (ppm) relative to TMS (0 ppm for $\left.{ }^{1} \mathrm{H}\right), \mathrm{CHCl}_{3}\left(77\right.$ ppm, for $\left.{ }^{13} \mathrm{C}\right)$, DMSO (39 ppm for ${ }^{13} \mathrm{C}$ ), and PhTeTePh (419 ppm in $\mathrm{CDCl}_{3}, 422 \mathrm{ppm}$ in DMSO for ${ }^{125} \mathrm{Te}$ ). Mass analyses were performed using a JEOL AccuTOF LC-plus JMS-T100LP spectrometer (Japan, Tokyo).

\section{2. (4-(Hydroxymethyl)Phenyl)(Phenyl)Telluride (2)}

A solution of diphenyl ditelluride $(81.9 \mathrm{mg}, 0.200 \mathrm{mmol}),(4-h y d r o x y m e t h y l) p h e n y l) b o r o n i c$ acid (1) (66.9 mg, $0.440 \mathrm{mmol}), 1,10$-phenthroline $\cdot \mathrm{H}_{2} \mathrm{O}(2.20 \mathrm{mg}, 12.0 \mathrm{~mol})$, and $\mathrm{CuSO}_{4}(19.0 \mathrm{mg}$, $12.0 \mathrm{~mol})$ in ethanol $(0.6 \mathrm{~mL})$ was stirred at room temperature for $1 \mathrm{~min}$. Then, a $5 \% \mathrm{Na}_{2} \mathrm{CO}_{3}$ aqueous solution $(0.1 \mathrm{~mL})$ was added to the solution and the mixture was stirred at room temperature for $5 \mathrm{~h}$. The progress of the reaction was monitored by ${ }^{1} \mathrm{H}$ NMR spectroscopy. After the reaction was completed, the mixture was dried over $\mathrm{MgSO}_{4}$, and the solvent was evaporated. The residue was purified via dry column chromatography to produce product $2(0.104 \mathrm{~g}, 84 \%)$ as a colorless solid. Mp 62-63 $\left.{ }^{\circ} \mathrm{C} ;{ }^{1} \mathrm{H}-\mathrm{NMR}\left(500 \mathrm{MHz}, \mathrm{CDCl}_{3}\right): \delta=7.69-7,67(\mathrm{~m}, 4 \mathrm{H}), \mathrm{J}=6,4 \mathrm{H}\right), 7.21-7.29(\mathrm{~m}, 5 \mathrm{H}), 4.66$ (s, 2H), 1.80 (br, 1H); ${ }^{13} \mathrm{C}-\mathrm{NMR}\left(125 \mathrm{MHz}, \mathrm{CDCl}_{3}\right): \delta=140.7,138.3,137.9,129.5,128.1,127.9,114.7$, 113.7, 65.0; ${ }^{125} \mathrm{Te}-\mathrm{NMR}\left(159 \mathrm{MHz}, \mathrm{CDCl}_{3}\right): \delta=690.7$; HRMS (APCI): $m / z[\mathrm{M}-\mathrm{OH}]^{+}$calcd. for $\mathrm{C}_{13} \mathrm{H}_{11} \mathrm{Te}$ 296.9917, found: 296.9874 .

\section{3. (4-(Chloromethyl)Phenyl)(Phenyl)Telluride (3)}

To a solution of (4-(hydroxymethyl)phenyl)(phenyl)telluride (2) (2.32 g, $7.45 \mathrm{mmol}$ ) and triethylamine $(1.50 \mathrm{~mL}, 10.4 \mathrm{mmol})$ in $\mathrm{CH}_{2} \mathrm{Cl}_{2}(4.6 \mathrm{~mL})$, a solution of thionyl chloride $(2.41 \mathrm{~mL}$, $33.5 \mathrm{mmol})$ in $\mathrm{CH}_{2} \mathrm{Cl}_{2}(7.4 \mathrm{~mL})$ was added dropwise at $0{ }^{\circ} \mathrm{C}$. The resulting mixture was stirred at room temperature for $1 \mathrm{~h}$. Then $\mathrm{CH}_{2} \mathrm{Cl}_{2}$ was removed by evaporation, THF $(36.9 \mathrm{~mL})$ and a $2 \mathrm{M} \mathrm{NaOH}$ solution $(25.2 \mathrm{~mL}$ ) were added, and the mixture was stirred at room temperature for $19 \mathrm{~h}$. After the reaction was completed, the mixture was evaporated and extracted with ethyl acetate. The organic layer was washed with water, dried over $\mathrm{MgSO}_{4}$, and evaporated. The residue was purified via column chromatography to yield product $3(2.32 \mathrm{~g}, 94 \%)$ as a yellow oil. ${ }^{1} \mathrm{H}-\mathrm{NMR}\left(500 \mathrm{MHz}, \mathrm{CDCl}_{3}\right)$ : $\delta=7.72(\mathrm{~d}, \mathrm{~J}=8.0,2 \mathrm{H}), 7.63(\mathrm{~d}, \mathrm{~J}=8.0,2 \mathrm{H}), 7.33-7.21(\mathrm{~m}, 5 \mathrm{H}), 4.55(\mathrm{~s}, 2 \mathrm{H}) ;{ }^{13} \mathrm{C}-\mathrm{NMR}\left(125 \mathrm{MHz} \mathrm{CDCl}_{3}\right)$ : $\delta=138.5,137.8,137.1,129.6,128.1$ (overlapped), 115.3, 114.3, 45.9; ${ }^{125} \mathrm{Te}-\mathrm{NMR}\left(159 \mathrm{MHz}, \mathrm{CDCl}_{3}\right)$ : $\delta=690.6$; HRMS (APCI): $m / z$ [M-Cl] ${ }^{+}$calcd. for $\mathrm{C}_{13} \mathrm{H}_{11}$ Te: 296.9917, found: 296.9951 .

\subsection{1-Methyl-3-(4-(Phenyltellanyl)Benzyl)-1H-Imidazol-3-Ium Chloride (4)}

A solution of phenyl(4-chloromethylphenyl)tellane (3) $(2.32 \mathrm{~g}, 7.02 \mathrm{mmol})$ and $\mathrm{N}$-methylimidazole $(0.692 \mathrm{~g}, 8.43 \mathrm{mmol})$ in $\mathrm{CH}_{3} \mathrm{CN}(63.6 \mathrm{~mL})$ was heated to reflux for $19 \mathrm{~h}$. Then, the mixture was evaporated, and the residue was purified via column chromatography to yield product $4(2.18 \mathrm{~g}, 75 \%)$ as a yellow oil. ${ }^{1} \mathrm{H}-\mathrm{NMR}\left(500 \mathrm{MHz}, \mathrm{DMSO}-d_{6}\right): \delta=9.42(\mathrm{~s}, 1 \mathrm{H}), 7.84(\mathrm{~s}, 1 \mathrm{H}), 7.75(\mathrm{~s}, 1 \mathrm{H}), 7.71(\mathrm{~d}, \mathrm{~J}=8.0,2 \mathrm{H}), 7.66(\mathrm{~d}, \mathrm{~J}=8.0$, $2 \mathrm{H}), 7.37-7.27$ (m, 5H), 5.45 (s, 2H), $3.86(\mathrm{~s}, 3 \mathrm{H}) ;{ }^{13} \mathrm{C}-\mathrm{NMR}\left(125 \mathrm{MHz}, \mathrm{DMSO}-d_{6}\right): \delta=138.7,137.9,137.24$, 135.0, 130.3, 129.9, 128.7, 124.8, 122.8, 116.6, 115.0, 51.8, 36.3; ${ }^{125} \mathrm{Te}-\mathrm{NMR}\left(159 \mathrm{MHz}, \mathrm{DMSO}-d_{6}\right): \delta=705.3$; HRMS (APCI): $m / z \mathrm{M}^{+}$calcd. for $\mathrm{C}_{17} \mathrm{H}_{17} \mathrm{~N}_{2}$ Te: 379.0448 , found: 379.0462 . 


\subsection{1-Methyl-3-(4-(Phenyltellanyl)Benzyl)-1H-Imidazol-3-Ium Hexafluorophosphate (5)}

A solution of 1-methyl-3-(4-(phenyltellanyl)benzyl)-1 $\mathrm{H}$-imidazol-3-ium chloride (4) (2.16 g, $5.24 \mathrm{mmol})$ and $\mathrm{KPF}_{6}(0.965 \mathrm{~g}, 5.24 \mathrm{mmol})$ in methanol $(10 \mathrm{~mL})$ was stirred at $30{ }^{\circ} \mathrm{C}$ for $17 \mathrm{~h}$. Then, the mixture was filtered, and the solution was dried over $\mathrm{MgSO}_{4}$ and evaporated to yield product $5(2.63 \mathrm{~g}, 96 \%)$ as a yellow oil. ${ }^{1} \mathrm{H}-\mathrm{NMR}\left(500 \mathrm{MHz}, \mathrm{DMSO}-d_{6}\right): \delta=9.18(\mathrm{~s}, 1 \mathrm{H}), 7.77-7.65$ $(\mathrm{m}, 6 \mathrm{H}), 7.38-7.26(\mathrm{~m}, 5 \mathrm{H}), 5.39(\mathrm{~s}, 2 \mathrm{H}), 3.84(\mathrm{~s}, 3 \mathrm{H}) ;{ }^{13} \mathrm{C}-\mathrm{NMR}\left(125 \mathrm{MHz}, \mathrm{DMSO}-d_{6}\right): \delta=138.7,137.9$, $137.2,134.9,130.3,129.9,128.7,124.5,122.8,116.6,115.0,51.9,36.3$; ${ }^{125} \mathrm{Te}-\mathrm{NMR}$ (159 MHz, DMSO- $\left.d_{6}\right)$ : $\delta=707.0$; HRMS (APCI): $m / z \mathrm{M}^{+}$calcd. for $\mathrm{C}_{17} \mathrm{H}_{17} \mathrm{~N}_{2}$ Te: 379.0448, found: 379.0491 .

\subsection{Oxidation of Thiols}

Typically, [bmim] $\mathrm{PF}_{6}$ solution $(10 \mathrm{~mL})$ of thiophenol $(0.110 \mathrm{~g}, 1.00 \mathrm{mmol})$, IL-supported diphenyl telluride $5(0.104 \mathrm{~g}, 0.200 \mathrm{mmol})$, and rose bengal $(50.9 \mathrm{mg}, 0.0500 \mathrm{mmol})$ were vigorously stirred in an open flask and irradiated using a 500-W halogen lamp for $3 \mathrm{~h}$. The resulting mixture was extracted with diethyl ether and evaporated to yield diphenyl disulfide $(0.114 \mathrm{~g}$, quant.). The catalytic oxidation of other thiols was similarly conducted and the products were identified by comparison of physical and spectral data with published values.

\subsubsection{Diphenyl Disulfide}

Pale pink solids, mp 55-56 ${ }^{\circ} \mathrm{C}\left(\mathrm{mp} 59-61{ }^{\circ} \mathrm{C}[14]\right) ;{ }^{1} \mathrm{H}-\mathrm{NMR}\left(500 \mathrm{MHz}, \mathrm{CDCl}_{3}\right): \delta=7.50(\mathrm{~d}, \mathrm{~J}=8.5$, $4 \mathrm{H}), 7.30(\mathrm{t}, \mathrm{J}=7.8,4 \mathrm{H}), 7.26(\mathrm{t}, \mathrm{J}=8.5,2 \mathrm{H}) ;{ }^{13} \mathrm{C}-\mathrm{NMR}\left(125 \mathrm{MHz}, \mathrm{CDCl}_{3}\right): \delta=137.0,129.1,127.5,127.2$.

\subsubsection{Didodecyl Disulfide}

Pale pink solids, $\mathrm{mp} 33-40{ }^{\circ} \mathrm{C}\left(\mathrm{mp} 31-32{ }^{\circ} \mathrm{C}[14]\right) ;{ }^{1} \mathrm{H}-\mathrm{NMR}\left(500 \mathrm{MHz}, \mathrm{CDCl}_{3}\right): \delta=2.70(\mathrm{t}, \mathrm{J}=7.5$, $4 \mathrm{H}), 1.68$ (quin., $\mathrm{J}=7.5,4 \mathrm{H}), 1.39(\mathrm{~m}, 4 \mathrm{H}), 1.26(\mathrm{br}, 32 \mathrm{H}), 0.88(\mathrm{t}, \mathrm{J}=6.8,6 \mathrm{H}) ;{ }^{13} \mathrm{C} \mathrm{NMR}(125 \mathrm{MHz}$, $\left.\mathrm{CDCl}_{3}\right): \delta=39.20,31.90,29.68,29.66,29.6029 .50,29.40,29.27,29.25,28.60,22.70,14.10$.

\subsubsection{Dicyclohexyl Disulfide}

Orange oil; ${ }^{1} \mathrm{H}-\mathrm{NMR}\left(500 \mathrm{MHz}, \mathrm{CDCl}_{3}\right): \delta=2.67-2.68(\mathrm{~m}, 2 \mathrm{H}), 2.03-2.05(\mathrm{~m}, 4 \mathrm{H}), 1.77-1.79(\mathrm{~m}$, $4 \mathrm{H}), 1.56-1.62(\mathrm{~m}, 2 \mathrm{H}), 1.25-1.31(\mathrm{~m}, 10 \mathrm{H}) ;{ }^{13} \mathrm{C} \mathrm{NMR}\left(125 \mathrm{MHz}, \mathrm{CDCl}_{3}\right): \delta=50.00,32.90,26.10,25.70$.

\subsubsection{Di-Tert-Butyl Disulfide}

Pink oil; ${ }^{1} \mathrm{H}-\mathrm{NMR}\left(500 \mathrm{MHz}, \mathrm{CDCl}_{3}\right): \delta=1.31(\mathrm{~s}, 18 \mathrm{H}) ;{ }^{13} \mathrm{C} \mathrm{NMR}\left(125 \mathrm{MHz}, \mathrm{CDCl}_{3}\right): \delta=46.20,30.60$.

\subsubsection{2-Hydroxyethyl Disulfide}

Pink oil; ${ }^{1} \mathrm{H}-\mathrm{NMR}\left(500 \mathrm{MHz}, \mathrm{CDCl}_{3}\right): \delta=3.92(\mathrm{t}, \mathrm{J}=5.8,4 \mathrm{H}), 2.89(\mathrm{t}, \mathrm{J}=5.8,4 \mathrm{H}) ;{ }^{13} \mathrm{C} \mathrm{NMR}$ $\left(125 \mathrm{MHz}, \mathrm{CDCl}_{3}\right): \delta=60.40,41.20$.

3.6.6. Dimethyl 3,3'-Dithiodipropionate

Pink oil; ${ }^{1} \mathrm{H}-\mathrm{NMR}\left(500 \mathrm{MHz}, \mathrm{CDCl}_{3}\right): \delta=3.71(\mathrm{~s}, 6 \mathrm{H}), 2.93(\mathrm{t}, \mathrm{J}=7.0,4 \mathrm{H}), 2.76(\mathrm{t}, \mathrm{J}=7.3,4 \mathrm{H}) ;{ }^{13} \mathrm{C}$ NMR (125 MHz, $\left.\mathrm{CDCl}_{3}\right): \delta=172.2,51.90,33.90,33.10$ [21].

\subsubsection{Dibenzyl Disulfide}

Colorless solids, $\mathrm{mp} 65-70{ }^{\circ} \mathrm{C}\left(\mathrm{mp} 70-71{ }^{\circ} \mathrm{C}[14]\right) ;{ }^{1} \mathrm{H}-\mathrm{NMR}\left(500 \mathrm{MHz}, \mathrm{CDCl}_{3}\right): \delta=7.29-7.33(\mathrm{~m}$, $4 \mathrm{H}), 7.26-7.28(\mathrm{~m}, 4 \mathrm{H}), 7.22-7.24(\mathrm{~m}, 2 \mathrm{H}), 3.59(\mathrm{~s}, 1 \mathrm{H}) ;{ }^{13} \mathrm{C} \mathrm{NMR}\left(125 \mathrm{MHz}, \mathrm{CDCl}_{3}\right): \delta=137.40,129.47$, $128.53,127.48,43.300$. 


\subsubsection{1,2-Bis(4-Nitrophenyl)Disulfane}

Yellow solids, mp 168-173 ${ }^{\circ} \mathrm{C}\left(\mathrm{mp} 178-180{ }^{\circ} \mathrm{C}[22]\right) ;{ }^{1} \mathrm{H}-\mathrm{NMR}\left(500 \mathrm{MHz}, \mathrm{CDCl}_{3}\right): \delta=8.20(\mathrm{~d}$, $J=9 \mathrm{~Hz}, 4 \mathrm{H}), 7.61(\mathrm{~d}, J=9 \mathrm{~Hz}, 4 \mathrm{H}) ;{ }^{13} \mathrm{C} \mathrm{NMR}\left(125 \mathrm{MHz}, \mathrm{CDCl}_{3}\right): \delta=147.30,144.38,126.70,124.79$.

\subsubsection{1,2-Bis(4-Chlorophenyl)Disulfane}

Colorless solids, mp 63-65 ${ }^{\circ} \mathrm{C}\left(\mathrm{mp} 65-66{ }^{\circ} \mathrm{C}[14]\right) ;{ }^{1} \mathrm{H}-\mathrm{NMR}\left(500 \mathrm{MHz}, \mathrm{CDCl}_{3}\right): \delta=7.39(\mathrm{~d}$, $J=8.5 \mathrm{~Hz}, 4 \mathrm{H}), 7.27(\mathrm{~d}, J=8.5 \mathrm{~Hz}, 4 \mathrm{H}) ;{ }^{13} \mathrm{C} \mathrm{NMR}\left(125 \mathrm{MHz}, \mathrm{CDCl}_{3}\right): \delta=135.44,133.94,131.08$.

\subsubsection{4,4'-Dipyridyl Disulfide}

Colorless solids, $\mathrm{mp} 59-64{ }^{\circ} \mathrm{C}\left(\mathrm{mp} 72-74{ }^{\circ} \mathrm{C}[23]\right) ;{ }^{1} \mathrm{H}-\mathrm{NMR}\left(500 \mathrm{MHz}, \mathrm{CDCl}_{3}\right): \delta=8.56-8.50(\mathrm{~m}$, $4 \mathrm{H}), 7.46-7.24(\mathrm{~m}, 4 \mathrm{H}) ;{ }^{13} \mathrm{C} \mathrm{NMR}\left(125 \mathrm{MHz}, \mathrm{CDCl}_{3}\right): \delta=150.4,149.9,121.8$.

\section{Conclusions}

We demonstrated the synthesis of the IL-supported organotelluride reagent and its application as a recyclable catalyst for thiol oxidation. A novel diphenyl telluride catalyst $\mathbf{5}$ carrying an imidazolium hexafluorophosphate moiety as an IL support was successfully synthesized. The synthesis was very easy, requiring only four steps. The IL-supported diphenyl telluride $\mathbf{5}$ was found to be an efficient catalyst for aerobic oxidation of various thiols under photosensitized conditions, affording the corresponding disulfides in excellent yields. Although the oxidation proceeds smoothly in various IL solutions, $[\mathrm{bmim}] \mathrm{PF}_{6}$ is best suited for efficient phase separation. The produced disulfides can be isolated and purified via simple extraction with diethyl ether. After removal of the product, the resulting IL solution containing IL-supported catalyst (5) and rose bengal can be reused at least four times without a considerable loss in catalytic activity (97\% yield in the fifth run).

Author Contributions: Conceptualization, S.K., and M.O.; synthetic methodology, A.M., S.K., and M.O.; investigation, M.M., Y.S., and A.M.; writing—original draft preparation, A.M.; writing—review and editing, A.M. All authors have read and agreed to the published version of the manuscript.

Funding: This research received no external funding.

Acknowledgments: We would like to thank Yoshiki Oda and Yoshimi Kanie for helpful inputs related to this work.

Conflicts of Interest: The authors declare no conflict of interest.

\section{References}

1. Petragnani, N.; Stefani, H.A. Tellurium in Organic Synthesis, 2nd ed.; Academic Press: London, UK, 2007; pp. 162-183.

2. Barton, D.H.R.; Ley, S.V.; Meerholz, C.A. Bis(p-methoxyphenyl) telluroxide: A new, mild oxidising agent. Chem. Commun. 1979, 755-756. [CrossRef]

3. Ley, S.V.; Meerholz, C.A.; Barton, D.H.R. Catalytic oxidation of thiocarbonyl compounds involving the use of 1,2-dibromotetrachloroethane as a brominating reagent for diaryl $\mathrm{Te}^{\mathrm{II}}$ species. Tetrahedron Lett. 1980, 21, 1785-1788. [CrossRef]

4. Ley, S.V.; Meerholz, C.A.; Barton, D.H.R. Diaryl telluroxides as new mild oxidising reagents. Tetrahedron 1981, 37, 213-223. [CrossRef]

5. Barton, D.H.R.; Finet, J.-P.; Thomas, M. Organotellurinic acid anhydrides as selective oxidants in organic synthesis. Tetrahedron 1986, 42, 2319-2324. [CrossRef]

6. Hu, N.X.; Aso, Y.; Otsubo, T.; Ogura, F. Polymer-supported diaryl selenoxide and telluroxide as mild and selective oxidizing agents. Bull. Chem. Soc. Jpn. 1986, 59, 879-884. [CrossRef]

7. Hu, N.X.; Aso, Y.; Otsubo, T.; Ogura, F. Novel oxidizing properties of $p$-methoxybenzenetellurinic acid anhydride. Tetrahedron Lett. 1986, 27, 6099-6102. [CrossRef]

8. Fukumoto, T.; Matsuki, T.; Hu, N.X.; Aso, Y.; Otsubo, T.; Ogura, F. Benzenetellurinic mixed anhydrides as mild oxidizing agents. Chem. Lett. 1990, 19, 2269-2272. [CrossRef] 
9. Kambe, N.; Tsukamoto, T.; Miyoshi, N.; Murai, S.; Sonoda, N. Oxidation of olefins with benzenetellurinic anhydride. Chem. Lett. 1987, 16, 269-272. [CrossRef]

10. Oba, M.; Endo, M.; Nishiyama, K.; Ouchi, A.; Ando, W. Photosensitized oxygenation of diaryl tellurides to telluroxides and their oxidizing properties. Chem. Commun. 2004, 14, 1672-1673. [CrossRef]

11. Oba, M.; Okada, Y.; Nishiyama, K.; Shimada, S.; Ando, W. Synthesis, characterization and oxidizing properties of a diorgano tellurone carrying bulky aromatic substituents. Chem. Commun. 2008, 5378-5380. [CrossRef]

12. Oba, M.; Okada, Y.; Nishiyama, K.; Ando, W. Aerobic photooxidation of phosphite esters using diorganotelluride catalysts. Org. Lett. 2009, 11, 1879-1881. [CrossRef] [PubMed]

13. Okada, Y.; Oba, M.; Arai, A.; Tanaka, K.; Nishiyama, K.; Ando, W. Diorganotelluride-catalyzed oxidation of silanes to silanols under atmospheric oxygen. Inorg. Chem. 2010, 49, 383-385. [CrossRef] [PubMed]

14. Oba, M.; Tanaka, K.; Nishiyama, K.; Ando, W. Aerobic oxidation of thiols to disulfides catalyzed by diaryl tellurides under photosensitized conditions. J. Org. Chem. 2011, 76, 4173-4177. [CrossRef] [PubMed]

15. Miao, W.; Chan, T.H. Ionic-liquid-supported synthesis: A novel liquid-phase strategy for organic synthesis. Acc. Chem. Res. 2006, 39, 897-908. [CrossRef]

16. Koguchi, S. An ionic-liquid-supported 18-crown-6 ether: Recyclable catalyst for acetylation and fluorination in anionic liquid. Trans. Mater. Res. Soc. Jpn. 2013, 38, 35-36. [CrossRef]

17. Koguchi, S.; Nakamura, K. Ascorbic acid based ionic liquid: Recyclable and efficient catalytic systems for the Huisgen cycloaddition. Synlett 2013, 24, 2305-2309. [CrossRef]

18. Koguchi, S.; Izawa, K. Ionic liquid-phase synthesis of 1,5-disubstituted 1,2,3-triazoles. ACS Comb. Sci. 2014, 16, 381-385. [CrossRef]

19. Koguchi, S.; Mihoya, A.; Mimura, M. Alcohol oxidation via recyclable hydrophobic ionic liquid-supported IBX. Tetrahedron 2016, 72, 7633-7637. [CrossRef]

20. Koguchi, S.; Shibuya, Y.; Igarashi, Y.; Takemura, H. Ionic liquid-supported 1,3-dimethylimidazolidin-2-one: Application as a reusable halogenation reagent. Synlett 2019, 30, 943-946. [CrossRef]

21. Dethe, D.H.; Srivastava, A.; Dherange, B.D.; Kumar, B.V. Unsymmetrical disulfide synthesis through photoredox catalysis. Adv. Synth. Catal. 2018, 360, 3020-3025. [CrossRef]

22. Kumar, V.; Kaushik, M.P. Efficient oxidative coupling of thiols into disulfides using N-tert-butyl-N-chlorocyanamide. Bull. Chem. Soc. Jpn. 2008, 81, 160-162. [CrossRef]

23. Kosobokov, M.; Cui, B.; Balia, A.; Matsuzaki, K.; Tokunaga, E.; Saito, N.; Shibata, N. Importance of a fluorine substituent for the preparation of meta- and para-pentafluoro- $\lambda^{6}$-sulfanyl-substituted pyridines. Angew. Chem. Int. Ed. 2016, 55, 10781-10785. [CrossRef] [PubMed] 